\title{
Status of artificial intelligence in Pakistan and its implications in anesthesiology
}

\author{
Muhammad Imran Khan ${ }^{1}$, Rana Shahzad Murtaza ${ }^{2} \square$, Muhammad Haider Ali $^{2} \square$, \\ Muhammad Sulaman Ashraf ${ }^{3} \bowtie$, Sahar Yazdani ${ }^{4} \bowtie$, Anum Yaseen ${ }^{5} \bowtie$
}

Author affiliation:

1. Postgraduate Resident Anesthesiology, Choudhary Pervaiz Elahi Institute of Cardiology, Multan, Pakistan.

2. Medical officer, Anesthesia, Choudhary Pervaiz Elahi Institute Of Cardiology, Multan, Pakistan.

3. Medical officer, Intensive Care, Choudhary Pervaiz Elahi Institute Of Cardiology, Multan, Pakistan.

4. Clinical Fellow, Tameside Hospital, Manchester, UK.

5. Medical Officer, Iqbal Hospital, Bahawalpur, Pakistan.

Correspondence: Dr Muhammad Imran Khan; E-mail: imrkhan803@gmail.com; Phone: +923326366121

\section{Abstract}

Artificial intelligence (AI) refers to the technology that enables machine-based intelligent behavior. This descriptive article gives an overview of the implementation of artificial intelligence in the following disciplines within the field of clinical medicine: Anesthesiology, Cardiology, Pulmonology, Endocrinology, Nephrology, Gastroenterology, Neurology, Histopathology, and Radiology. In this article, we will discuss the employment of artificial intelligence in anesthesiology and provide an analysis of the several areas in which it is being utilized. We will also evaluate the current status of $\mathrm{Al}$ and its future in Pakistan. The importance of this manuscript is to highlight the benefits of artificial intelligence so that the benefits can be realized in Pakistan.

Key words: Artificial intelligence; Anesthesiology; Clinical medicine; Endocrinology; Gastroenterology; Histopathology; Nephrology; Neurology; Pakistan; Radiology

Citation: Khan MI, Murtaza RS, Ali MH, Ashraf MS, Yazdani S, Yaseen A. Status of artificial intelligence in Pakistan and its implications in anesthesiology. Anaesth. pain intensive care 2022;26(1):110-114; DOI:

10.35975/apic.v26i1.1776

Received: September 12, 2021, Reviewed: November 24, 2021, Accepted: December 10, 2021

\section{Introduction}

Artificial intelligence (AI) has altered the way many firms operate by assisting them in doing their tasks more effectively. ${ }^{1} \mathrm{AI}$ is basically a computer program that is capable of performing tasks typically associated with human intelligence, e.g. complicated problem solving, decision making, object detection, etc. AI makes use of the complex blend of mathematics and computer science. AI can provide outcomes that are more accurate than that of human beings. ${ }^{2}$ AI performs more convenient work because it doesn't need any break and refreshment time compared to the human beings. It constantly performs its work for long time duration without facing any problem. There are no factors influencing the performance of AI as opposed to factors which influence the work of human beings. ${ }^{3}$

\section{Background}

Alan Turing created the "Turing test" in 1950 to determine if a computer could do a task indistinguishably from that of a person. After the 1990s, several AI approaches were used in the healthcare sector, including Bayesian networks, fuzzy expert systems, artificial neural networks, and deep learning, etc. ${ }^{4}$ After the successful deployment of AI methods in a variety of sectors including healthcare, a significant investment was made specifically for the development of AI-based technologies for the healthcare sector. 
The use of AI in medicine is divided into two parts. One part is physical and includes all robotic applications which can assist during surgeries, early diagnosis, and treatment of handicapped patients. ${ }^{5}$ While the other element is virtual, which includes all programs that assist experts in making treatment decisions, such as massive recorded data systems analysis using neural networks.

\section{Implementation of $\mathbf{A l}$ in healthcare system}

The implementation of AI-based techniques gives a power to healthcare professionals to search for appropriate solutions for emerging clinical problems. ${ }^{6}$ Healthcare sectors have a huge amount of medical data that are gathered from several areas of the medical sector, data that can easily be utilized by a deep learning algorithm. Currently, there are some departments of clinical medicine that use AI-based techniques and get advantages from them. ${ }^{7}$ Some examples include: use of ECG in the detection of atrial fibrillation, detection of electrical activity in brain (like during epileptic seizures), detection of the sugar level in the blood, and detection of disease, related to deep tissue examination through imaging techniques in radiology, anesthesia, intensive care we well as interventional pain management. ${ }^{8}$ This advanced technology helps healthcare professionals to indulge in the concept of personalized treatment. ${ }^{9}$

\section{Application of Al in medicine}

The following section discusses current uses of AI in medicine. ${ }^{10}$

\subsection{Cardiology}

AI transformed medicine for the first time in 2014 when it was used for the identification of atrial fibrillation using digital ECG monitoring equipment. These remote device applications made it possible to identify arrhythmias. Apple's wristwatch capable of detecting atrial fibrillation has been cleared by the FDA. However, despite this, there are some limitations to this approach, such as the false positive rate that is shown to the public ${ }^{9}$. Through AI-based techniques like deep learning and neural network, machines are able to detect a risk of cardiovascular disease in people with high accuracy. ${ }^{10}$

\subsection{Pulmonology}

In pulmonary function testing, AI plays a critical role by allowing software specialists to more accurately interpret the test results and proceed with subsequent treatment procedures. $^{11}$

\subsection{Endocrinology}

Numerous businesses have obtained FDA permission to produce a smart monitoring gadget for diabetes patients that enables frequent monitoring of blood glucose levels. $^{12}$

\subsection{Nephrology}

AI also helps in the treatment of kidney disease by detecting the rate of filtration in the patient and also predicts the risk of mortality related to nephrology. ${ }^{13}$

\subsection{Gastroenterology}

Deep learning and neural network approaches of AI system help in the endoscopy and ultrasound procedure by aiding in the diagnosis of precancerous and cancerous lesions so as to facilitate the patient's treatment by earlier detection of problematic lesions. ${ }^{14}$.

\subsection{Neurology}

Electroencephalography that use AI to identify epileptic episodes in patients. This gadget utilizes the most advanced technology available for improving seizure therapy via reliable monitoring. Gadgets like BIS, NIRS etc. powered by AI are critical for objectively detecting problems in neurological patients. ${ }^{15}$

\subsection{Histopathology}

There is a big challenge for a healthcare professional to recognize cancer cells in patients. By the implementation of an AI-based algorithm, the professionals are able to find the cancerous tissue with a high accuracy rate and successfully remove the tumor margin. ${ }^{16}$

\subsection{Radiology}

Radiologists used a deep learning approach in imagedbased diagnosis. Studies are done on making appropriate models through CT scanner, MRI, and x-ray for nextgeneration radiologist tool development. ${ }^{17}$

\section{Role of Al in anesthesia}

The term "Anesthesiology" refers to all measures that are concerned before, after, and also during the patient surgical operations. In its perioperative preparations includes giving anesthesia, critical care medicine, emergency treatment, and pain management. In today's advanced technological period, when many areas profit from AI-based solutions, AI computers can oversee the job of anesthesiologist autonomously. ${ }^{18}$ There are different areas of anesthesiology where AI techniques are utilized.

\subsection{Measurement of depth of anesthesia}

Several studies have shown improvement in the measurement of depth of anesthesia by using AI. Especially in electroencephalography testing, it shows brain activity when the brain cells communicate through electrical impulses to find out the depth of anesthesia at the time of BIS monitoring. The use of machine learning AI helps to analyze a complex range of 
electroencephalography signals to evaluate the effects of certain drugs in patients. ${ }^{19}$

\subsection{Standard control over anesthesia delivery}

AI helps to construct a standard closed-loop anesthesia delivery system with real-time monitoring. According to this, the anesthesiologist used an automated medication administration strategy to ensure the patient's safety by maximizing clinical indicators or markers that indicate the level of anesthesia. Apart from blood pressure as a vital indicator, BIS measurement using AI is being used to establish control over anesthetic administration. To facilitate the endotracheal intubation a deep machine learning approach is used to deliver the neuromuscular blockade automatically. ${ }^{20-22}$

\subsection{Complication event prediction}

There are many approaches based on AI, such as machine learning, neural networks, and fuzzy logic that are being used to assess the possibility of danger occurrence through hypnotic effect assessment. Throughout surgery, a fuzzy neural network can automatically assess the patient's awareness after general anesthetic, assisting in decision-making and adjusting anesthetic during the procedure. There are two locations where one must identify event occurrences: the intensive care unit (ICU) and the operating room. ${ }^{21}$ Here, machine learning is used to evaluate a vast database in order to forecast any event, such as morbidity, mortality, or sepsis.

\subsection{Assist ultrasound-based practice}

The neural network approach from an AI-based network is most commonly used to assist the ultrasound-based practice by its accurate imaging features. ${ }^{22}$ By using this technique professionals easily distinguish between the two images' differences. Additionally, these approaches aid in identifying anatomical levels for the administration of anesthetic from the spinal cord insertion in ultrasound imaging.

\subsection{Pain management services}

AI specialists control discomfort during preoperative measurements in order to aid patients. By using a deep learning method, professionals were able to evaluate MRI data in order to distinguish between painful and non-painful stimuli. ${ }^{23}$ Machine learning is also used to analyze the photoplethysmography and skin conductance wave data of patients. Machine learning also applies for detecting the patient who responds to acute pain for therapy. ${ }^{23}$

\subsection{Management of operating room}

AI also plays a role in evaluating the aspects affecting operating room logistics, such as time management in OT or sensing an anesthesiologist's activities. This technique used large amounts of electronic data and a trained neural network method to forecast the overall duration of the surgical operation, as well as the kind of surgery procedure ${ }^{24}$. Tags were examined using machine learning to monitor anesthesiologists' interactions with operating room equipment.

\section{Current status of $\mathrm{Al}$ in Pakistan}

Numerous industries use AI in their operations on a worldwide scale. Pakistan is also developing into a significant market for AI-based solutions. Pakistan uses AI technology in a variety of industries to provide better answers to existing and emerging challenges and to boost demand in the new age. ${ }^{25}$ Pakistan enhanced its selfsecurity systems as a result of this, including missiles powered by AI, cyber security, and very effective cameras. There has been a noticeable increase in the spectrum of AI applications in Pakistan during the last several years.

\section{PIAIC project in Pakistan}

To involve the youth generation the Pakistan government initiated the PIAIC project for the development and progress of the country's economy. The government develops national-level AI centers around the country. ${ }^{26}$ Currently, commercial companies in Pakistan are using $\mathrm{AI}$ in their operations by engaging top AI professionals. The following AI laboratories are located in Pakistan:

- The National University of Sciences \& Technology (NUST) - National Center of AI, Islamabad.

- Artificial intelligence research lab (AIRL) Alkhawarizmi Institute of Computer Science (KICS), University of Engineering \& Technology (UET), Lahore.

- The Center of Intelligent Systems and Network Research (CISNR) University of Engineering \& Technology (UET), Peshawar.

- The Artificial Intelligence Lab, The Institute of Business Administration IBA (The AI Lab), Karachi

Pakistan has to restructure its faculty in the area of medicine to include AI-based learning and also take initiatives to improve the appeal of new students to hightechnology learning approaches aimed at transforming the medical sector via technological services. Implementation of these approaches would catapult Pakistan's economy to new heights. ${ }^{26}$ 


\section{The Future of $\mathrm{Al}$ in Pakistan}

AI has a significant influence on the economic growth of several nations worldwide. Pakistan, on the other hand, is making relatively modest progress in implementing AI applications in a variety of disciplines, including medical. Several educational institutions and corporate companies, for example, NUST University, have introduced this AI course and department in order to compete in the market. Pakistan's agricultural department also employs a machine learning strategy to deal with natural disasters in the country. ${ }^{27}$

\section{Conclusion}

In conclusion, the deployment of AI-based approaches improves the precision of executing diverse tasks. AI assists healthcare providers by transforming the field of medicine with its accurate prediction capabilities. In clinical medicine, AI-based approaches are applied in a variety of fields, including anesthesiology. This article discussed numerous areas of anesthesiology in which AIbased solutions are being used to improve patient outcomes. Finally, we noted that Pakistan is a long way from reaping the benefits of this industry. Pakistan's healthcare industry is unlikely to benefit from this breakthrough in the future without further developing an expertise in AI.

\section{Conflict of interest}

The author declares no conflict of interest.

\section{Authors contribution}

All authors took part in literature search and drafting and review of this manuscript.

\section{References}

1. Davenport TH, Ronanki R. Artificial intelligence for the real world. Harvard Bus Rev. 2018;96(1):108-116. [Free Full Text]

2. Acemoglu D, Restrepo P. 8. Artificial Intelligence, Automation, and Work. In: Agrawal A, Gans J, Goldfarb A (ed.) The Economics of Artificial Intelligence: An Agenda. Chicago: University of Chicago Press; 2019. p.197-236. DOI: 10.7208/9780226613475-010

3. Wu H, Han H, Wang X, Sun S. Research on artificial intelligence enhancing internet of things security: a survey. IEEE Access. 2020;8:153826-153848. 10.1109/ACCESS.2020.3018170

4. Amisha, Malik P, Pathania M, Rathaur VK. Overview of artificial intelligence in medicine. J Family Med Prim Care. 2019 Jul;8(7):2328-2331. [PubMed] DOI: 10.4103/jfmpc.jfmpc_440_19
5. Lorkowski J, Grzegorowska O, Pokorski M. Artificial Intelligence in the healthcare system: an overview. Adv Exp Med Biol. 2021;1335:1-10. [PubMed] DOI: 10.1007/5584_2021_620

6. Morley J, Floridi L. An ethically mindful approach to Al for health care. Lancet. 2020 Jan 25;395(10220):254-255. [PubMed] DOI: 10.1016/S0140-6736(19)32975-7

7. Panch $T$, Szolovits $P$, Atun R. Artificial intelligence, machine learning and health systems. J Glob Health. 2018 Dec;8(2):020303. [PubMed] DOI: 10.7189/jogh.08.020303

8. Briganti G. Augmented Medicine: Changing Clinical Practice with Artificial Intelligence. In: Advances in Artificial Intelligence, Computation, and Data Science. Springer, Cham; 2021. p. 333339. DOI: 10.1007/978-3-030-69951-2_14

9. Becker A. Artificial intelligence in medicine: What is it doing for us today? Health Policy Tech. 2019;8(2):198-205. DOI: 10.1016/j.hlpt.2019.03.004

10. Dorado-Díaz P, Sampedro-Gómez J, Vicente-Palacios V, Sánchez P. Applications of artificial intelligence in cardiology. the future is already here. Revista Española de Cardiología. 2019;72(12):1065-1075. 10.1016/j.rec.2019.05.014

[PubMed]

DOI:

11. Das N, Topalovic M, Janssens W. Artificial intelligence in diagnosis of obstructive lung disease. Curr Opin Pulm Med. 2018;24(2):117-123. [PubMed] DOl: 10.1097/MCP.0000000000000459

12. Dvey-Aharon $Z$, Huhtinen $P$. Screening for diabetic retinopathy in endocrinology clinics by using handheld cameras and applying artificial intelligence algorithms. J Endocrine Soc. 2021;5(Suppl 1):A419-A425. DOI: $10.1210 \% 2$ Fjendso\%2Fbvab048.856

13. Niel O, Bastard P. Artificial intelligence in nephrology: core concepts, clinical applications, and perspectives. Am J Kidney Dis. 2019;74(6):803-810. [PubMed] DOI: 10.1053/j.ajkd.2019.05.020

14. Yang YJ, Bang CS. Application of artificial intelligence in gastroenterology. World J Gastroenterol. 2019 Apr 14;25(14):1666-1683. [PubMed] DOl: 10.3748/wjg.v25.i14.1666

15. Raghavendra U, Acharya UR, Adeli H. Artificial intelligence techniques for automated diagnosis of neurological disorders. Eur Neurol. 2019;82(1-3):41-64. [PubMed] DOI: $10.1159 / 000504292$

16. Sultan AS, Elgharib MA, Tavares T, Jessri M, Basile JR. The use of artificial intelligence, machine learning and deep learning in oncologic histopathology. J Oral Pathol Med. 2020 Oct;49(9):849-856. [PubMed] DOI: 10.1111/jop.13042

17. Y Yasaka K, Abe O. Deep learning and artificial intelligence in radiology: Current applications and future directions. PLoS Med. 2018 Nov 30;15(11):e1002707. [PubMed] DOI: 10.1371/journal.pmed.1002707

18. Hashimoto DA, Witkowski E, Gao L, Meireles O, Rosman G. Artificial intelligence in anesthesiology: current techniques, clinical applications, and limitations. Anesthesiology. 2020 Feb;132(2):379-394. [PubMed] DOI: 10.1097/ALN.0000000000002960

19. Lönnqvist PA. Asleep or awake: is paediatric regional anaesthesia without general anaesthesia possible? $\mathrm{Br} \mathrm{J}$ 
Anaesth. 2020 Aug;125(2):115-117. [PubMed] DOI: 10.1016/j.bja.2020.04.071

20. Wingert T, Lee C, Cannesson M. Machine learning, deep learning, and closed loop devices-anesthesia delivery. Anesthesiol Clin. 2021 Sep;39(3):565-581. [PubMed] DOI: 10.1016/j.anclin.2021.03.012

21. Xia M, Xu T, Jiang $H$. Progress and perspective of artificial intelligence and machine learning of prediction in anesthesiology. J Shanghai Jiaotong Uni (Sci). 2021;1-9. DOI: 10.1007/s12204-021-2331-3

22. Bowness J, El-Boghdadly K, Burckett-St Laurent D. Artificial intelligence for image interpretation in ultrasound-guided regional anaesthesia. Anaesthesia. 2021 May;76(5):602-607. [PubMed] DOI: 10.1111/anae.15212

23. Wang R, Wang S, Duan N, Wang Q. From patient-controlled analgesia to artificial intelligence-assisted patient-controlled analgesia: practices and perspectives. Front Med (Lausanne).
2020 May 22;7:145. [PubMed] DOI: 10.3389/fmed.2020.00145

24. Bellini V, Guzzon M, Bigliardi B, Mordonini M, Filippelli S, Bignami E. Artificial intelligence: a new tool in operating room management. role of machine learning models in operating room optimization. J Med Syst. 2019 Dec 10;44(1):20. [PubMed] DOI: 10.1007/s10916-019-1512-1

25. Shahid MZ, Li G. Impact of artificial intelligence in marketing: a perspective of marketing professionals of Pakistan. Global J Manag Bus Res. 2019;19(2-E). [Free Full Text]

26. Jamil S. Artificial intelligence and journalistic practice: The crossroads of obstacles and opportunities for the Pakistani journalists. Journalism Pract. 2020;15(10):1400-1422. DOI: 10.1080/17512786.2020.1788412

27. Abid S, Awan B, Ismail T, Sarwar N, Sarwar G, Tariq M, et al. Artificial intelligence: medical student $\mathrm{S}$ attitude in district Peshawar Pakistan. PJPH. 2019;9(1):19-21. DOI: 10.32413/pjph.v9i1.295 